HEAT TRANSFER (WILEY, USA)

Editor: Dr. William M. Worek, Texas A \&M University, Kingsville, USA.

Online ISSN:2688-4542

Accepted September $29^{\text {th }} 2020$

\title{
COMPUTATION OF RADIATIVE MARANGONI (THERMOCAPILLARY) \\ MAGNETOHYDRODYNAMIC CONVECTION IN CU-WATER BASED NANOFLUID FLOW FROM A DISK IN POROUS MEDIA: SMART COATING SIMULATION
}

\section{Shamshuddin ${ }^{*}$}

${ }^{1}$ Department of Mathematics, Vaagdevi College of Engineering (Autonomous), Warangal, Telangana, India.

Email: shammaths@gmail.com,shamshuddin_md@vaagdevi.edu.in

\section{S.R. Mishra ${ }^{2}$}

${ }^{2}$ Department of Mathematics, Siksha O Anusandhan Deemed to be University, Bhubaneswar, India.

Email: satyaranjan_mshr@yahoo.co.in

\section{O. Anwar Bég ${ }^{3}$}

${ }^{3}$ Professor and Director-Multi-Physical Engineering Sciences Group, Mechanical Engineering Department, School of Science, Engineering and Environment (SEE), University of Salford, Manchester, UK.

Email: gortoab@gmail.com, O.A.Beg@salford.ac.uk

\section{Tasveer. A. Bég ${ }^{4}$}

${ }^{4}$ Director, Engineering Mechanics Research, Israfil House, Dickenson Rd., Manchester, UK.

Email: tasveerabeg@gmail.com

\section{Ali Kadir 5}

${ }^{5}$ Department of Mechanical/Aeronautical Engineering, Salford University, Manchester, M54WT, UK.

Email: A.Kadir@salford.ac.uk 


\begin{abstract}
With emerging applications for smart and intelligent coating systems in energy, there has been increasing activity in researching magnetic nano nanomaterial coating flows. Surface tension features significantly in such regimes, and in presence of heat transfer, Marangoni (thermocapillary) convection arises. Motivated by elaborating deeper the intrinsic transport phenomena in such systems, in this paper, a mathematical model is developed for steady radiative heat transfer and Marangoni magnetohydrodynamic (MHD) flow of $\mathrm{Cu}$-water nanofluid under strong magnetic field from a disk adjacent to a porous medium. The semi-analytical Adomain Decomposition Method (ADM) is employed to solve the governing equations which are reduced into ordinary differential equation form via the Von Karman similarity transformation. Validation with a GDQ (Generalized Differential Quadrature) algorithm is included. The response in dimensionless velocity, temperature, wall heat transfer rate and shear stress is investigated for various values of the control parameters. Temperature is reduced with increasing Marangoni parameter whereas the flow is accelerated. With increasing permeability parameter temperatures are elevated. Increasing radiative flux boosts temperatures further from the disk surface. Increasing magnetic parameter strongly damps the boundary layer flow and elevates the temperatures, also eliminating temperature oscillations at lower magnetic field strengths.
\end{abstract}

KEYWORDS: Magnetohydrodynamics; Thermal radiation, Marangoni flow, Nanofluid, ADM, Nano-coating flows; Boundary layers; Volume fraction; Porous Media; GDQ computation.

\title{
1. INTRODUCTION
}

Marangoni flows in electrically conducting liquids produce many intriguing applications. In such flows the transport (carrier) liquid responds to external magnetic fields and invokes magnetohydrodynamic effects. In this sense the system is "smart" and can respond to external stimuli. Ma et al. [1] observed that electromagnetic damping of the forced convection exceeds that due to buoyant convection and an intricate interplay exists between magnetic body force, thermal buoyancy and Marangoni surface tension. Further studies in this area were communicated by Langlois et al. [2] for finite electrical conductivity and weak magnetic field and Lin et al. [3] for power-law non-Newtonian magnetic liquids. Rudraiah et al. [4] employed an Alternating Direction Implicit (ADI) method for non-linear convective terms (with upwind differencing) and Successive Line Over Relaxation (SLOR) algorithm for low Prandtl number $(\mathrm{Pr}=0.54)$ (in a rectangular open cavity). Outcomes of this investigation identified that at large, two counter rotating cells are formed at upper and lower half of the enclosure for (Grashof number range- $2 \times 10^{4}$ to $2 \times 10^{6}$, Marangoni number range- 0 to $10^{5}$ and Hartmann number range- 0 
to 100). They further showed that with increasing magnetic field (Hartmann number), the temperature field streamlines are extended in the horizontal direction and the temperature field assumes that of thermal conduction. They also reported that average Nusselt number is elevated with Marangoni number but depressed with Hartmann number. Jana et al. [5] used a finite element method to simulate Silicon crystal-melt interface and oxygen transport in magnetohydrodynamic Marangoni convection, by modelling the crystal-melt interface according to Stefan's balance via grid displacement. They observed that increasing axial magnetic field suppresses interface topology, reduces oxygen concentration levels and furthermore induces non-uniformity in oxygen distribution at the crystalmelt interface. Zueco and Anwar Bég [6] employed PSPICE electrothermal software to study the free and forced convection in Magnetohydrodynamic Marangoni flow with a thermocapillary coupling condition $\mathrm{n}$ included in the interfacial boundary conditions at the interface. They showed that greater Hartmann hydromagnetic number retards the boundary layer flow whereas it increases thermal boundary layer thickness. The converse effect is found to be induced for the case of assistive Marangoni flow. Witkowski and Walker [7] investigated axisymmetric flow driven by Marangoni convection and a rotating magnetic field (RMF) in a crystal growth floating-zone regime, at low Prandtl number, observing that the flow field evolves from a Marangoni dominated flow to a RMF dominated flow with stronger magnetic field and examining the transition region (at which sources of motion eliminate each other) in detail. Hajabdollahi and Premnath [8] studied the Marangoni convection in heated liquid pools formed during melting under strong magnetic field. They derived novel similarity solutions for an imposed interfacial nonlinear heat flux variation, noting that with increasing power-law exponent there are more distinct variations in the surface heat flux profiles and more intense Marangoni convection whereas the contrary response is induced with stronger magnetic field (deceleration and damping).

Radiative heat transfer in above studies are neglected which is also of interest in high-temperature materials processing, coating synthesis etc. [9]. Radiation is the most complex of heat transfer modes and most simulations deploy algebraic flux models to avoid an explicit solution of the general nonlinear integro-differential radiative equation. Milne-Eddington approximation, Schuster-Schwartzchild two-flux model, Rosseland flux model and Hamaker 6-flux model are examples of flux models. Anwar Bég et al. [10] used a network electrothermal code and non-linear Rosseland diffusion approximation Rosseland's diffusion flux model to simulate the radiative Von Karman slip flow from a rotating disk under strong transverse magnetic field. Ganesh et al. [11] used a shooting method and nonlinear Rosseland flux model to study radiative Marangoni boundary layer convection of $\gamma \mathrm{Al}_{2} \mathrm{O}_{3}$ nanofluids along a linear stretching sheet with Ethylene glycol $\left(\mathrm{C}_{2} \mathrm{H}_{6} \mathrm{O}_{2}\right)$ and Water $\left(\mathrm{H}_{2} \mathrm{O}\right)$ as base fluids. They noted that greater nanoparticle volume fraction parameter suppresses flow acceleration whereas greater radiative 
flux and nanoparticle volume fraction parameter elevates the local Nusselt number. Lin et al. [12] computed the radiative Marangoni convection flow and heat transfer in pseudo-plastic non-Newtonian nanofluids with $\mathrm{Cu}$, $\mathrm{Al}_{2} \mathrm{O}_{3}, \mathrm{CuO}$ and $\mathrm{TiO}_{2}$ nanoparticles and sodium carboxymethyl cellulose (CMC)-water used as base fluid. Mahanthesh et al. [13] presented numerical solutions for Marangoni transport of dissipating SWCNT and MWCNT nanofluids under the influence of magnetic force and radiation with an exponential space dependent heat source, observing that Nusselt number is elevated with Marangoni convection whereas it is suppressed with magnetic field. Bayazitoglu and Lam [14] investigated the impact of radiative transfer on hydrodynamic stability of Marangoni convection in a Newtonian fluid layer of infinite horizontal extent confined between a free upper surface and a rigid isothermal lower surface. They employed radiative boundaries of black-black, mirror-mirror, and black-mirror type and a perturbation method based on calculus of variations. They noted that in reduced gravity conditions (e.g. spacecraft environments) stronger radiation flux suppresses Marangoni convection.

In the present work, we extend the earlier model of Lin and Zheng [15] to consider magnetohydrodynamic body force and radiative flux effects. The conservation equations are rendered into dimensionless form via the Von Karman transformations. An interfacial boundary condition is implemented to simulate Marangoni (surface tension) convection effects and we employed Adomain Decomposition Method (ADM) [16] for the proposed model. Extensive visualization of the impact of nano-particle solid volume fraction, Marangoni number, magnetic body force number, Prandtl number, permeability parameter and radiative parameter on flow rate and energy is done. Also, we have presented fluid wall friction, temperature gradient and Sherwood number for diverse quantities. The results are validated by benchmark results of GDQ (Generalized Differential Quadrature) method [17] which are tabulated and achieved excellent correlation. The model presented herein therefore applies to hightemperature smart electroconductive coatings [18-20]. Two-dimensional, laminar steady viscous radiative magnetohydrodynamic flow of copper -hydrogen oxide (water) nanofluid coating flow over an infinite circular disk inserted in a porous medium is studied. The Darcy model is employed to analyze the bulk matrix drag effect. This model has been utilized successfully in previous computational materials coating simulations [21-23] and is valid for viscous-dominated flows in homogenous, isotropic permeable media. The Tiwari-Das nanoscale model [24] is employed which allows variation of nano-particle volume fraction to be studied for specific nanoparticles and in this regard the Tiwari-Das formulation is superior to the Buongiorno two-component nanoscale model [25]. The Tiwari-Das model is highly amenable to numerical simulations and has also been applied very recently in solar nanofluid collector systems [26]. The simulations are relevant to nanofluid magnetic fuel cells and smart electroconductive coating systems. 


\section{THERMO-CAPILLARY MAGNETIC RADIATIVE COATING FLOW MODEL}

We consider two-dimensional, axisymmetric, laminar steady viscous radiative convection flow of magnetic coating $\mathrm{Cu}$-Water nanoliquid over an infinite solid impervious disk embedded in a saturated porous medium. Flow is mobilized by a temperature gradient induced via surface tension (thermocapillary effect). A transverse static magnetic field is imposed. Unidirectional radiative flux is present and the coating nanoliquid is assumed to be optically dense [10]. Figure. 1 visualizes the flow regime in a cylindrical coordinate system. Marangoni surface tension effects act as a boundary condition on the governing equations of the flow field. The relevant mass, momentum and energy conservation equations are obtained by adding magnetic body force and radiative flux terms to the Lin-Zheng model [15]:

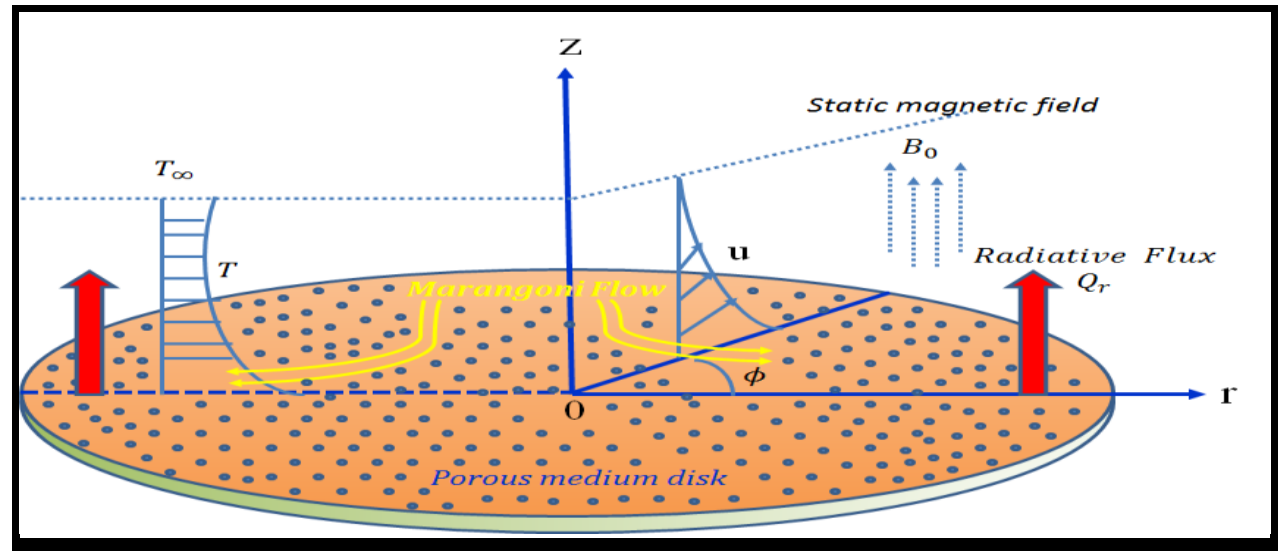

Figure 1: Physical model diagram for Marangoni hydromagnetic radiative nanofluid flow from a disk in a porous medium

$\frac{\partial u}{\partial r}+\frac{u}{r}+\frac{\partial w}{\partial z}=0$

$u \frac{\partial u}{\partial r}+w \frac{\partial u}{\partial z}=\frac{\mu_{n f}}{\rho_{n f}} \frac{\partial^{2} u}{\partial z^{2}}-\frac{\mu_{n f}}{\rho_{n f}} \frac{u}{K}-\frac{\sigma_{n f} B_{0}^{2}}{\rho_{n f}} u$,

$u \frac{\partial T}{\partial r}+w \frac{\partial T}{\partial z}=\frac{k_{n f}}{\left(\rho c_{p}\right)_{n f}} \frac{\partial^{2} T}{\partial z^{2}}-\frac{1}{\left(\rho c_{p}\right)_{n f}} \frac{\partial q_{r}}{\partial z}$,

The boundary conditions at the disk surface and in the free stream are:

at $z=0: \quad \mu_{n f} \frac{\partial u}{\partial z}=\frac{\partial \sigma}{\partial r}, w=0, T=T_{0}=T_{\infty}+A^{*} r^{2}$

at $z \rightarrow \infty: u=0, T=T_{\infty}$

Here $(u, v)$ are velocities along with $(r, z)$ directions. $\mu_{n f}$ denotes dynamic viscosity of nanofluid, $\rho_{n f}$ denotes density of nanofluid, $\sigma_{n f}$ denotes electrical conductivity of nanofluid, $k_{n f}$ denotes thermal conductivity 
of nanofluid, $\left(\rho c_{p}\right)_{n f}$ heat capacity of nanofluid, $\sigma^{*}$ denotes Stefan-Boltzmann constant, $\sigma$ denotes the surface tension,$k^{*}$ denotes mean absorption coefficient, $T$ denotes temperature of nanofluid, $T_{\infty}$ denotes ambient temperature and $T_{0}$ denotes temperature of nanofluid on the disk, $A^{*}$ is temperature constant. $\tau=\mu_{n f}(\partial u / \partial z)$ is shear stress. Further, it is assumed that the surface tension varies linearly with the temperature such that $\sigma=\sigma_{0}-\gamma_{T}\left(T-T_{\infty}\right)$ where $\gamma_{T}=-(\partial \sigma / \partial T)_{T=T_{\infty}}$. Here $\sigma_{0}, \gamma_{T}$ are positive constants which respectively represent the surface tension when $T=T_{\infty}$ and temperature coefficient of surface tension. $q_{r}$ signifies approximated Rosseland heat radiative flux and is assumed from [27(new add)] and which is denoted as $\frac{\partial q_{r}}{\partial z}=-\frac{16 \sigma^{*}}{3 k^{*}} \frac{\partial T^{4}}{\partial z^{2}}$, Also, $\mu_{n f}$ is approximated as the viscosity of the base fluid with $\mu_{f}$ containing a dilute suspension of the fine spherical particles, following [28] and they are defined as

$$
\begin{aligned}
& \mu_{n f}=\mu_{f}(1-\phi)^{-2.5}, \rho_{n f}=\rho_{f}(1-\phi)+\phi \rho_{s},\left(\rho c_{p}\right)_{n f}=\left(\rho c_{p}\right)_{f}(1-\phi)+\phi\left(\rho c_{p}\right)_{s}, \\
& \alpha_{n f}=\frac{k_{n f}}{\left(\rho c_{p}\right)_{n f}}, \frac{k_{n f}}{k_{f}}=\frac{\left(k_{s}+2 k_{f}\right)-2 \phi\left(k_{f}-k_{s}\right)}{\left(k_{s}+2 k_{f}\right)+\phi\left(k_{f}-k_{s}\right)}
\end{aligned}
$$

Where $\phi$ is solid volume fraction of the nanofluid, $\rho_{s}$ is the density of nanofluid, $\rho_{f}$ is the density of the base fluid, $\left(\rho c_{p}\right)_{s}$ the heat capacity of the nanoparticle, $k_{f}$ is thermal conductivity of the base fluid, $k_{s}$ is thermal conductivity of the nanoparticle. Invoking the Von Karman similarity transformations:

$u=r \Omega F(\xi), \quad w=\sqrt{\Omega v_{f}} H(\xi), T=T_{\infty}+A^{*} r^{2} \theta(\xi), \quad \xi=z \sqrt{\frac{\Omega}{v_{f}}}$

Inserting (5)-(6) into equations (1)-(4), we get the following two-point boundary value problem

$$
\begin{aligned}
& F+\frac{1}{2} H^{\prime}=0 \\
& F^{\prime \prime}-A\left[F^{2}+H F^{\prime}\right]-F[P+C M]=0 \\
& \frac{1}{\operatorname{Pr}}(1+R) \theta^{\prime \prime}-B\left[H \theta^{\prime}+2 F \theta\right]=0 \\
& F^{\prime}=-2 M a C, \quad H=0, \quad \theta=1 \quad \text { at } \xi=0 \\
& F=0, \quad \theta=0 \quad \text { at } \xi \rightarrow \infty
\end{aligned}
$$

Here prime denotes derivatives with respect to $\xi$ and $F, H$ are dimensionless velocities, $\Omega$ is a frequency $(1 / \mathrm{s})$.

$$
\begin{aligned}
& \tau(\xi)=-(1 / 2 C) F^{\prime}(\xi) \text { is dimensionless shear stress (note that } \tau=\mu_{n f} \frac{\partial u}{\partial z}=\frac{1}{C} \Omega r \mu_{f} \sqrt{\frac{\Omega}{\gamma_{f}}} F^{\prime}(\xi), \\
& \tau(\xi)_{\xi=0}=-M a \text { ) and } \theta \text { is dimensionless temperature. Here }
\end{aligned}
$$


$A=\left[(1-\phi)+\phi \frac{\rho_{s}}{\rho_{f}}\right](1-\phi)^{2.5}, B=\left[(1-\phi)+\phi \frac{\left(\rho c_{p}\right)_{s}}{\left(\rho c_{p}\right)_{f}}\right] \frac{\left(\frac{k_{s}}{k_{f}}+2\right)+\phi\left(1-\frac{k_{s}}{k_{f}}\right)}{\left(\frac{k_{s}}{k_{f}}+2\right)-2 \phi\left(1-\frac{k_{s}}{k_{f}}\right)}, C=(1-\phi)^{2.5} \quad$ and the other parameters are $P=\frac{v_{f}}{K \Omega}$ (permeability parameter), $M=\frac{\sigma_{f} B_{0}^{2}}{\rho_{f} \Omega}$ (magnetic body force parameter), $\operatorname{Pr}=\frac{v_{f}\left(\rho c_{p}\right)_{f}}{k_{f}}$ (Prandtl number), $R=\frac{16 \sigma^{*} T_{\infty}^{3}}{3 k_{f} k^{*}}$ (radiation parameter) and $M a=\frac{\gamma_{T} A *}{\Omega \mu_{f}} \sqrt{\frac{v_{f}}{\Omega}}$ (Marangoni parameter). The local Nusselt number $N u_{x}$ is given by:

$N u_{x}=-r \frac{\left\{-\left(\frac{\partial T}{\partial z}\right)_{z=0}+\left(q_{r}\right)_{z=0}\right\}}{[T(x, 0)-T(x, \infty)]}$

Dimensionless Nusselt number can be written as follows which provides a quantification of the heat transfer rate at the disk surface.

$$
\frac{N u_{x}}{\sqrt{\operatorname{Re}_{x}}}=-\left(\frac{k_{n f}}{k_{f}}+R\right) \sqrt{\frac{\Omega}{\gamma_{f}}} \theta^{\prime}(0)
$$

\section{ADM SOLUTION AND VALIDATION WITH GDQ}

For the system of coupled ordinary differential equations (7)-(9) with respect to boundary conditions (10) constitute a nonlinear, multi-degree fifth order differential boundary value problem. Numerous methods are available to solve such systems including shooting methods, finite element methods etc. Here a semianalytical/numerical method called the Adomain Decomposition Method (ADM) [29] is employed. In ADM, the analytic approximate solutions to a nonlinear equation are obtained without linearization and discretization yielding more accurate results. ADM has been deployed recently in a variety of complex multi-physical fluid dynamic problems including squeeze film magnetic bio-lubrication [30], stagnation flow of nanofluids on rotating bodies [31], magnetically actuated ciliated propulsion [32] and swirling bioconvection nanofluid from a rotating radially stretching disk [33]. Aski et al., [29] utilized recursive relations and also produced an alternative approach. The following standard procedure

$$
L u(y)+R u(y)+N u(y)=Q(y)
$$

which is derived from the form $D\{u)\}=Q(y)$. Here $D$ signifies differential operator of linear term $\{L+R\}$

with $L$ being the highest ordered derivative which is easily invertible and $R$ is the remaining linear part. The nonlinear part is $N u(y)$. It follows that the standard form can be written as follows: 
$u(y)=L^{-1}\{Q(y)\}-L^{-1}\{R u(y)\}-L^{-1}\{N u(y)\}$

Next we introduce $L_{1}=d^{3} / d \xi^{3}()$ and $L_{2}=d^{3} / d \xi^{3}()$ with invertible operators $L_{1}^{-1}()=\int_{0}^{\xi} \int_{0}^{\xi} \int_{0}^{\xi}() d \xi d \xi d \xi$ and $L_{2}^{-1}(\quad)=\int_{0}^{\xi} \int_{0}^{\xi}() d \xi d \xi$. Thus, arranging all the Eqns. (7)-(9) and by writing them as infinite series using recursive relations with initial guesses, the approximate analytical solutions can be obtained for the variables (velocities, $F, H$ and temperature $\theta$ ). For further details, the reader is referred to [33]. The ADM power series expansions with notations are as follows:

$$
\begin{aligned}
F(\xi)= & -\frac{1}{2} H^{\prime}(\xi) \\
H(\xi)= & \theta_{0}(\xi)+\theta_{1}(\xi)+\theta_{2}(\xi)+\theta_{3}(\xi) \\
H(\xi)= & \alpha \xi-M a c \xi^{2}+T_{1} \xi^{3}+T_{2} \xi^{4}+T_{3} \xi^{5}+T_{4} \xi^{6} \\
& +\left(T_{5}+T_{6}\right) \xi^{7}+T_{7} \xi^{8}+T_{8} \xi^{9}+T_{9} \xi^{10} \\
\theta(\xi)= & H_{0}(\xi)+H_{1}(\xi)+H_{2}(\xi)+H_{3}(\xi) \\
\theta(\xi)= & 1+\beta \xi+T_{10} \xi^{2}+T_{11} \xi^{3}+\left(T_{12}+T_{13}\right) \xi^{4}+T_{14} \xi^{5} \\
& +\left(T_{15}+T_{17}\right) \xi^{6}+\left(T_{16}+T_{18}\right) \xi^{7}+T_{19} \xi^{8}+T_{20} \xi^{9}+T_{21} \xi^{10}
\end{aligned}
$$

Where:

$$
\begin{aligned}
& H_{0}(\xi)=\alpha \xi-M a c \xi^{2}, \\
& \theta_{0}(\xi)=1+\beta \xi
\end{aligned}
$$




$$
\begin{aligned}
& T_{1}=\frac{1}{6}\left\{\frac{1}{2} \alpha^{2} A-\alpha(P+C M)\right\} ; T_{2}=\frac{1}{12} M a C(P+C M) ; T_{3}=\frac{1}{20}\left\{T_{1} \alpha A+T_{1}(P+C M)\right\} ; \\
& T_{4}=\frac{1}{60}\left\{-4 T_{2} \alpha A+9 M a C A-2 T_{2}(P+C M)\right\} ; T_{5}=\frac{3}{35} T_{2} M a C A ; \\
& T_{6}=\frac{1}{210}\left\{T_{3} \alpha A-\frac{21}{2} T_{1}^{2} A-5 T_{3}(P+C M)\right\} ; \\
& T_{7}=\frac{1}{356}\left\{-24 T_{4} \alpha A+32 T_{3} M a C A-6 T_{1} T_{2} A-6(P+C M) T_{4}\right\} ; \\
& T_{8}=\frac{1}{504}\left\{-35 T_{5} \alpha A+50 T_{4} M a C A-4 T_{2}^{2} A-7 T_{5}(P+C M)\right\} ; \\
& T_{9}=\frac{1}{720}\left\{70 T_{5} M a C A+2 T_{5} M a C A\right\} ; T_{10}=-\frac{1}{2}\left\{\frac{\operatorname{Pr} B}{1+R} \alpha\right\} ; \\
& T_{11}=\frac{1}{3}\left\{\frac{\operatorname{Pr} B}{1+R} M a C\right\} ; T_{12}=\frac{1}{12}\left\{\frac{\operatorname{Pr} B}{1+R} M a C \beta\right\} ; T_{13}=\frac{1}{12}\left\{\frac{\operatorname{Pr} B}{1+R}\left(T_{10} \alpha-3 T_{1}\right)\right\} ; \\
& T_{14}=\frac{1}{20} \frac{\operatorname{Pr} B}{1+R}\left\{2 T_{11} \alpha-2 T_{1} \beta-4 T_{2}\right\} ; T_{15}=\frac{1}{30} \frac{\operatorname{Pr} B}{1+R}\left\{3 T_{12} \alpha-T_{11} M a C-3 T_{2} \beta\right\} ; \\
& T_{16}=-\frac{1}{21} \frac{\operatorname{Pr} B}{1+R}\left\{T_{12} M a C\right\} ; T_{17}=\frac{1}{30} \frac{\operatorname{Pr} B}{1+R}\left\{3 T_{13} \alpha-T_{1} T_{10}-5 T_{3}\right\} ; \\
& T_{18}=\frac{1}{42} \frac{\operatorname{Pr} B}{1+R}\left\{4 T_{14} \alpha-2 T_{13} M a C+T_{1} T_{12}-T_{2} T_{11}-6 T_{4} \beta-7 T_{5}\right\} ; \\
& T_{19}=\frac{1}{56} \frac{\operatorname{Pr} B}{1+R}\left\{5 T_{15} \alpha-3 T_{14} M a C+T_{1} T_{12}-T_{2} T_{11}-6 T_{4} \beta-7 T_{5}\right\} ; \\
& T_{20}=\frac{1}{72} \frac{\operatorname{Pr} B}{1+R}\left\{6 T_{16} \alpha-4 T_{15} M a C-6 T_{5} \beta\right\} ; T_{21}=-\frac{1}{90} \frac{\operatorname{Pr} B}{1+R}\left\{5 T_{16} M a C\right\} .
\end{aligned}
$$

Novel correlation is reported with specific standardized numerical method called general differential quadrature method as no existing solutions available to validate. The detail procedure of GDQ available in the literature [34] which has been deployed recently in variety of complex multi-physical fluid dynamic problems including [3536]. It has also been used recently in nanofluids dynamics [34]. Benchmark present solutions which are presented in Table 1. Table 1 displays comparative study of $F(0), \theta^{\prime}(0)$ for different $\phi, M a, M, P, \operatorname{Pr}, R$. With increasing Marangoni number $(M a)$, there is an acceleration in the flow thus temperature is boosted. These results agree with

Lin and Zheng [15]. As $M$ increases, there is also a significant deceleration in the flow (due to the Lorentzian magnetic drag) whereas the heat transfer gradient is increased. This result was also obtained by other investigators including Hajabdollahi and Premnath [8]. An elevation in permeability parameter, $P$ (decreasing porous media permeability), results in greater Darcian resistance to the flow and induces retardation; however, it leads to a strong enhancement in wall temperature gradient. With increasing Prandtl number (Pr) and radiation parameter $(R)$, there is no tangible modification in the velocity field; however the temperature significantly increased with 
higher values of $\operatorname{Pr}$ whereas it is markedly suppressed with greater $R$ (since heat transport to the disk surface is inhibited and temperatures are increased within the nanofluid due to energization from the radiative flux).

Table 1: $F(0), \theta^{\prime}(0)$ comparison for different values of $\phi, M a, M, P, \operatorname{Pr}, R$

\begin{tabular}{|c|c|c|c|c|}
\hline$\phi$ & $F(0)(\mathrm{ADM})$ & GDQ & $\theta^{\prime}(0)(\mathbf{A D M})$ & GDQ \\
\hline 0 & 0.0452 & 0.0450 & -1.4865 & -1.4861 \\
\hline 0.015 & 0.0389 & 0.0386 & -1.4097 & -1.4094 \\
\hline 0.02 & 0.0374 & 0.0371 & -1.3875 & -1.3872 \\
\hline 0.03 & 0.0347 & 0.0343 & -1.3472 & -1.3471 \\
\hline \multicolumn{5}{|l|}{ Ma } \\
\hline 0.4 & -0.0026 & -0.0023 & -1.4556 & -1.4551 \\
\hline 0.3 & 0.012 & 0.0109 & -1.4077 & -1.4072 \\
\hline 0.2 & 0.0408 & 0.0411 & -1.4334 & -1.4331 \\
\hline 0.1 & 0.0855 & 0.0852 & -1.5497 & -1.5492 \\
\hline \multicolumn{5}{|l|}{$\mathbf{M}$} \\
\hline 0.1 & -0.0575 & -0.0572 & -0.0575 & -0.0572 \\
\hline 0.3 & -0.2135 & -0.2133 & 1.4473 & 1.44732 \\
\hline 0.5 & -0.2846 & -0.2841 & 3.1935 & 3.19347 \\
\hline 0.7 & -0.358 & -0.3577 & 5.3 & 5.2911 \\
\hline \multicolumn{5}{|l|}{$\mathbf{P}$} \\
\hline 0 & -0.1766 & -0.1764 & 0.7489 & 0.7486 \\
\hline 0.2 & -0.2511 & -0.2509 & 2.3207 & 2.3204 \\
\hline 0.4 & -0.3245 & -0.3242 & 4.3126 & 4.3124 \\
\hline 0.6 & -0.4025 & -0.4021 & 6.6547 & 6.6543 \\
\hline \multicolumn{5}{|l|}{ Pr } \\
\hline 0.7 & -0.1766 & -0.1764 & -0.2067 & -0.2069 \\
\hline 1 & -0.1766 & -0.1764 & -0.1813 & -0.1817 \\
\hline 4 & -0.1766 & -0.1764 & 0.2381 & 0.2388 \\
\hline 7 & -0.1766 & -0.1764 & 0.7489 & 0.7485 \\
\hline \multicolumn{5}{|l|}{$\mathbf{R}$} \\
\hline 1 & -0.2148 & -0.2146 & 0.5148 & 0.5144 \\
\hline 2 & -0.2148 & -0.2146 & 0.2266 & 0.2261 \\
\hline 3 & -0.2148 & -0.2146 & 0.0924 & 0.0927 \\
\hline 4 & -0.2148 & -0.2146 & 0.0164 & 0.0168 \\
\hline
\end{tabular}

\section{DISCUSSION OF RESULTS}

Figures. 2- 15 indicates velocity and temperature distribution responses to variation in the key control parameters, obtained via ADM solutions. Figure 2 illustrates the evolution in velocity with $\phi$. Generally, a decrease in velocity is induced such investigations can be found by other researchers including Ganesh et al., [11]. There is a weak reverse in this effect far from the disk surface, however. Overall an absence of nanoparticles ( $\phi=0$ i.e. pure water) achieves the best flow acceleration due to a lower overall viscosity whereas the highest volume fraction with $\mathrm{Cu}$-water nanofluid $(\phi=3 \%)$ is associated with the strongest retardation throughout boundary layer. Backflow is not induced anywhere in the boundary layer on the disk. Figure 3 shows that there is a clear elevation in temperature in the nanofluid with increasing volume fraction, although this is principally localized in the vicinity of the disk and the effect is depleted towards the free stream. Of course, relatively low volume fraction has been 
studied i.e. up to 3\%. It is envisaged that higher volume fraction of copper nanoparticles would also continue to enhance temperatures, although there is an upper limit to this trend i.e. at much higher volume fractions, the doping becomes counter-productive and inhibits thermal diffusion [37] and experimentally confirmed by Choi [38]. Figure 4 indicates that increasing $\phi$ weakly decreases the shear stress at the disk surface, which is again consistent with the flow deceleration computed in Fig. 3). The effect is sustained at all values of the coordinate, $\xi$. Figure 5 describes the impact of increasing Marangoni term to corresponding variations in velocity. A significant acceleration in the boundary layer flow is generated with greater values of $M a$. Marangoni convection is associated with surface tension at the surface of the nanofluid and depends on temperature. $M a=\frac{\gamma_{T} A * \sqrt{\frac{v_{f}}{\Omega}}}{\Omega \mu_{f}}$ and higher $M a$ values imply greater surface tension contribution. This reduces temperatures but increases velocity magnitudes. Figure 6 illustrates temperature magnitudes are depressed with increasing Marangoni number i.e. temperature decreases as the surface tension increases, and this observation is consistent with many other studies in this area including [14-15]. Figure 7 describes a weak decrease in surface shear stress is induced with increasing Marangoni number, $M a$. Figure 8 portray a strong resistance to the flow velocity for a rising values of $(M)$. The parameter $M=\sigma_{f} B_{0}^{2} / \rho_{f} \Omega$ relates the Lorentzian magnetic drag force to the inertial force in the flow. $M$ arises in the linear impedance term in the momentum eqn. (10). For $M=0$ the nanofluid is electrically non conducting and the magnetic field exerts no influence. As $M$ increases the contribution of magnetic Lorentzian force progressively increases and this induces retardation in the flow. At higher values of $M(0.5,0.7)$ negative velocity is created close to the disk surface, although this effect is eliminated at higher values of the coordinate, $\xi$. Excellent flow control is achieved therefore with an external magnetic field whereas significant flow acceleration is generated with increasing Marangoni effect. To regulate the flow on the disk therefore a careful selection of these two effects is required by designers. Figure $\mathbf{9}$ for varying values of magnetic field $(M)$ term. The term $(M)$ raised the heat transport in the system because the induced Lorentz force damped the flowing fluid which in turn stimulates viscosity and encourages energy source terms. As a result, energy distribution is improved and heat is able to transfer quickly from the disk surface. Figure $\mathbf{1 0}$ shows that the shear stress at the disk surface is generally increased with increasing magnetic parameter, except at very high values of the coordinate, $\xi$. The influence of $P$ (Permeability) on velocity, temperature and disk surface shear stress is illustrated in Figures. 1113. The parameter, $P=v_{f} / K \Omega$ and is inversely proportional to the actual permeability of the porous medium adjacent to the disk. This parameter arises in the linear Darcian drag force term in the momentum eqn. (10). For 
higher values of $P$, the permeability is lower and the Darcian drag force is naturally greater (more solid fibers are present to resist the flow). This induces strong deceleration in the boundary layer flow as seen in Figure 11. The presence of a porous medium may therefore be exploited to damp the flow significantly in hydromagnetic Marangoni convection. Reverse flow can also be amplified close to the disk surface (zero and small values of $\xi$ ). This backflow is in fact present even for the purely nanofluid regime $(P=0)$ but is exacerbated with decreasing permeability of the porous medium (i.e. more intense packing of fibers). Figure $\mathbf{1 2}$ shows that temperatures are accentuated strongly with increasing values of $P$. The progressive decrease in permeability (and corresponding elevation in Darcian impedance) encourages thermal conduction in the porous medium. Although the nanofluid cannot percolate as easily at higher values of $P$, this is compensated for via tighter packing of fibers (decreasing sparsity) which enables improved heat conduction through the porous medium, as noted by [39-40]. Although in the present simulations, a Darcian model has been adopted, with isotropic considerations, future studies may explore orthotropic media as studied earlier by Anwar Bég et al. [41] i.e. wherein permeability varies in both radial and tangential directions. Figure $\mathbf{1 3}$ shows that with increasing permeability parameter there is initially a reduction in shear stress at the disk surface; however, with further distance from the disk surface this trend is reversed and thereafter returns to the initial behavior in the free stream. For the purely nanofluid case $(\mathrm{P}=0)$ there is a steady monotonic growth in shear stress with $\xi$-coordinate; however, for non-zero $\mathrm{P}$ values, the profiles demonstrate an undulatory nature. Overall lower permeability successfully damps the flow in the vicinity of the disk, and this further testifies to the excellent flow control properties of porous media. Figure $\mathbf{1 4}$ visualizes the temperature evolution in the regime with a change in Prandtl number, $\operatorname{Pr}$. The monotonic decay computed for $\operatorname{Pr}<1$ and $\operatorname{Pr}=1$ is replaced with a temperature overshoot for $\operatorname{Pr}>1$. As $\operatorname{Pr}$ term rise, the thermal boundary layer is weakened, which leads to rising heat dispersion to the ambiances. This thereby shrinks the quantity of heat in the system and causes a consistent drop in the heat field as presented in the plot. Fig. 15 indicates that a significant boost in temperatures in the nanofluid is induced with an increase in radiative parameter, $R . R=16 \sigma^{*} T_{\infty}^{3} / 3 k f^{k^{*}}$ and relates the relative contribution of radiative heat transfer to that of conduction heat transfer. It is also known as the Stark number and Rosseland number. Presence of radiative heat flux energizes the magnetic nanoliquid regime and prominently elevates temperatures further from the disk surface since supplementary heat is added to the nanoliquid. Radiative term exerts marked ascendancy on heat transfer characteristics for slight modification, although in the near-wall zone temperatures are initially suppressed, again due to the presence of Marangoni convection. In the absence of thermal radiation, $R \rightarrow 0$ and this produces maximum temperatures near the disk 
surface (with an associated overshoot); however deeper into the boundary layer the contrary behavior is induced, and significant heating arises. A similar pattern has been computed by Ganesh et al., [11]. The neglection of radiative effects in mathematical models therefore severely under-predicts actual temperatures in the zone further from the disk surface and over-predicts temperatures closer to the disk surface.

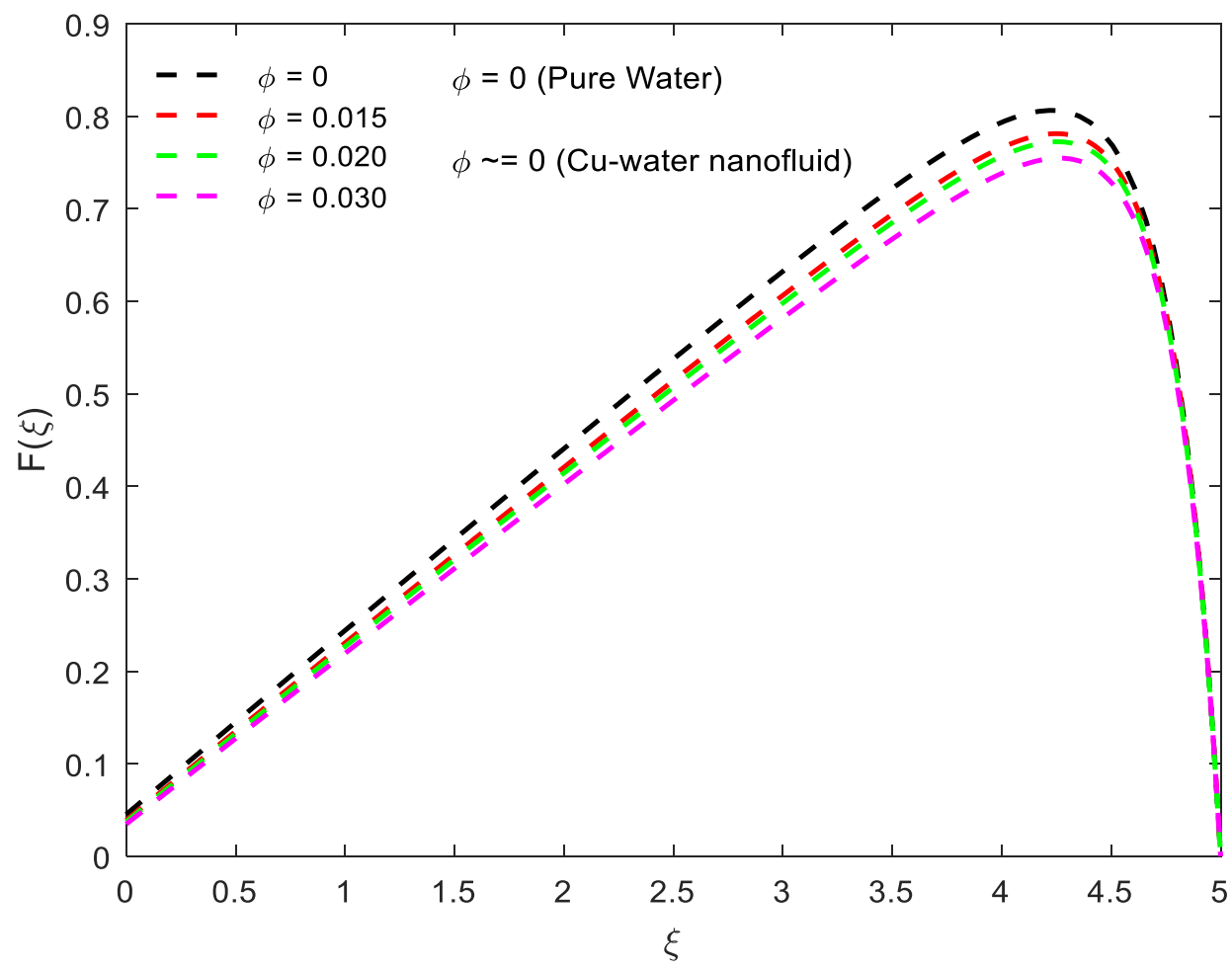

Figure 2: Volume fraction influence on $F(\xi)$ for $M=0, M a=0.2, P=0, R=0, \operatorname{Pr}=7, \phi=0$

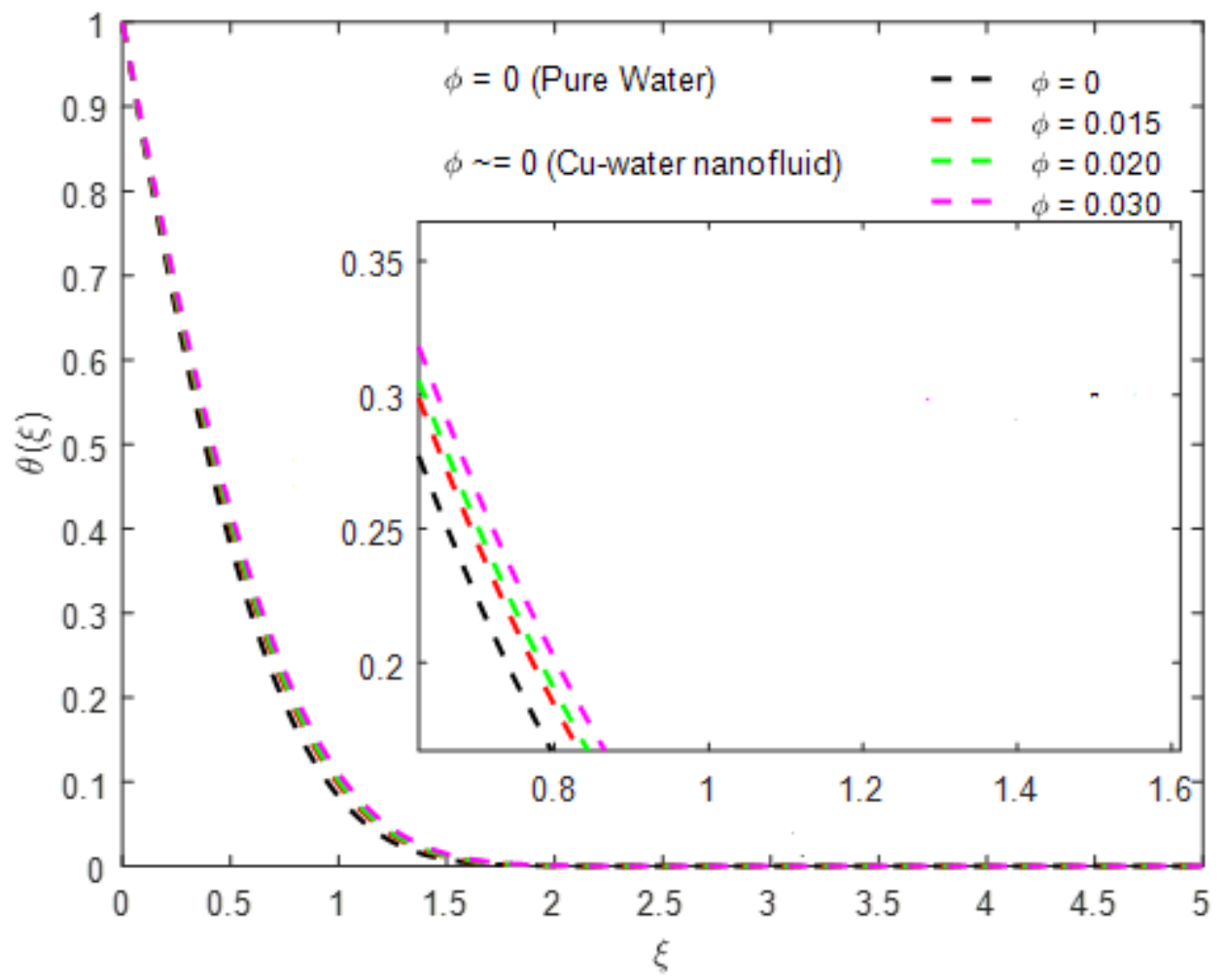

Figure 3: Volume fraction influence on $\theta(\xi)$ for $M=0, M a=0.2, P=0, R=0, \operatorname{Pr}=7, \phi=0$ 


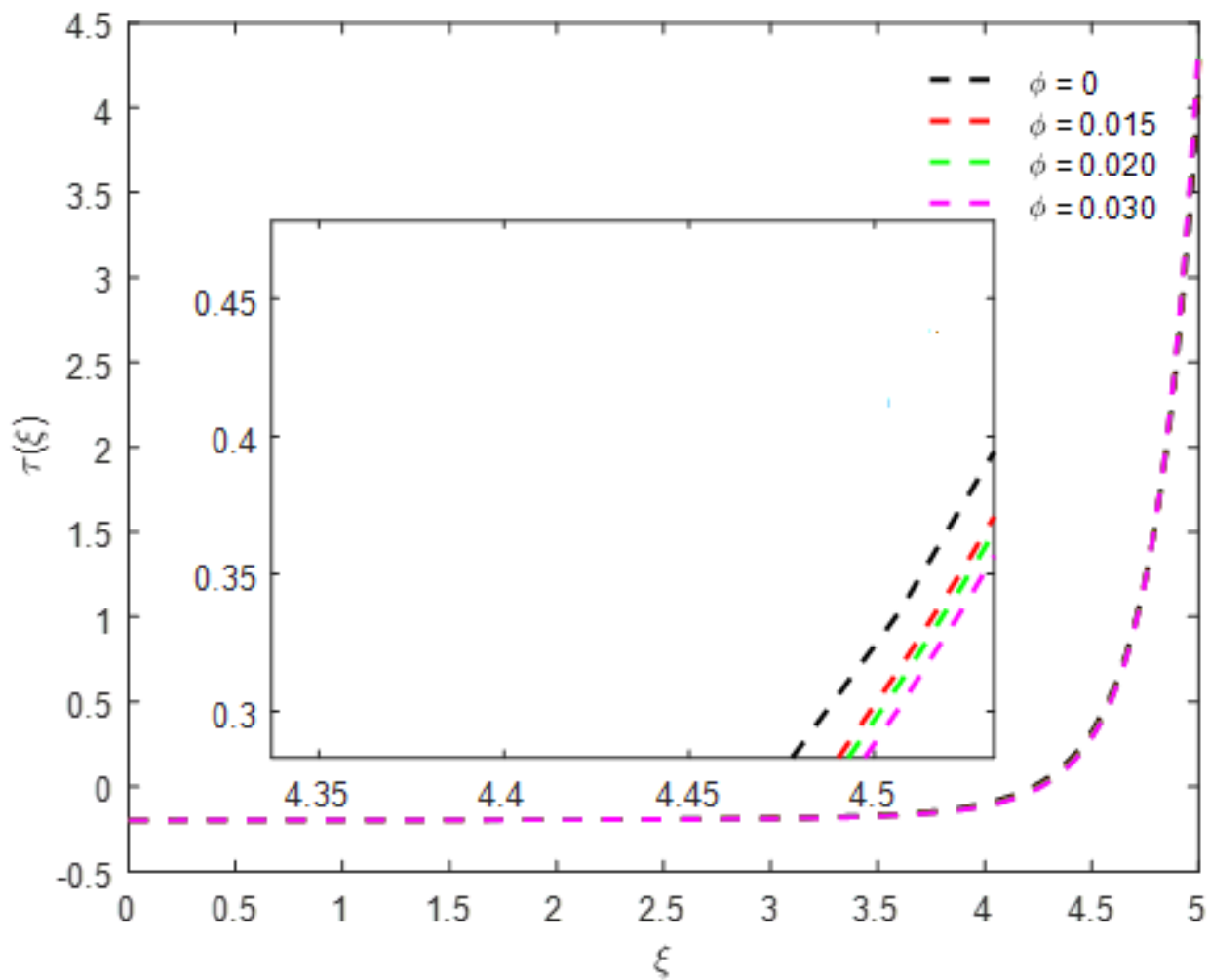

Figure 4: Volume fraction influence on $\tau(\xi)$ for $M=0, M a=0.2, P=0, R=0, \operatorname{Pr}=7, \phi=0$

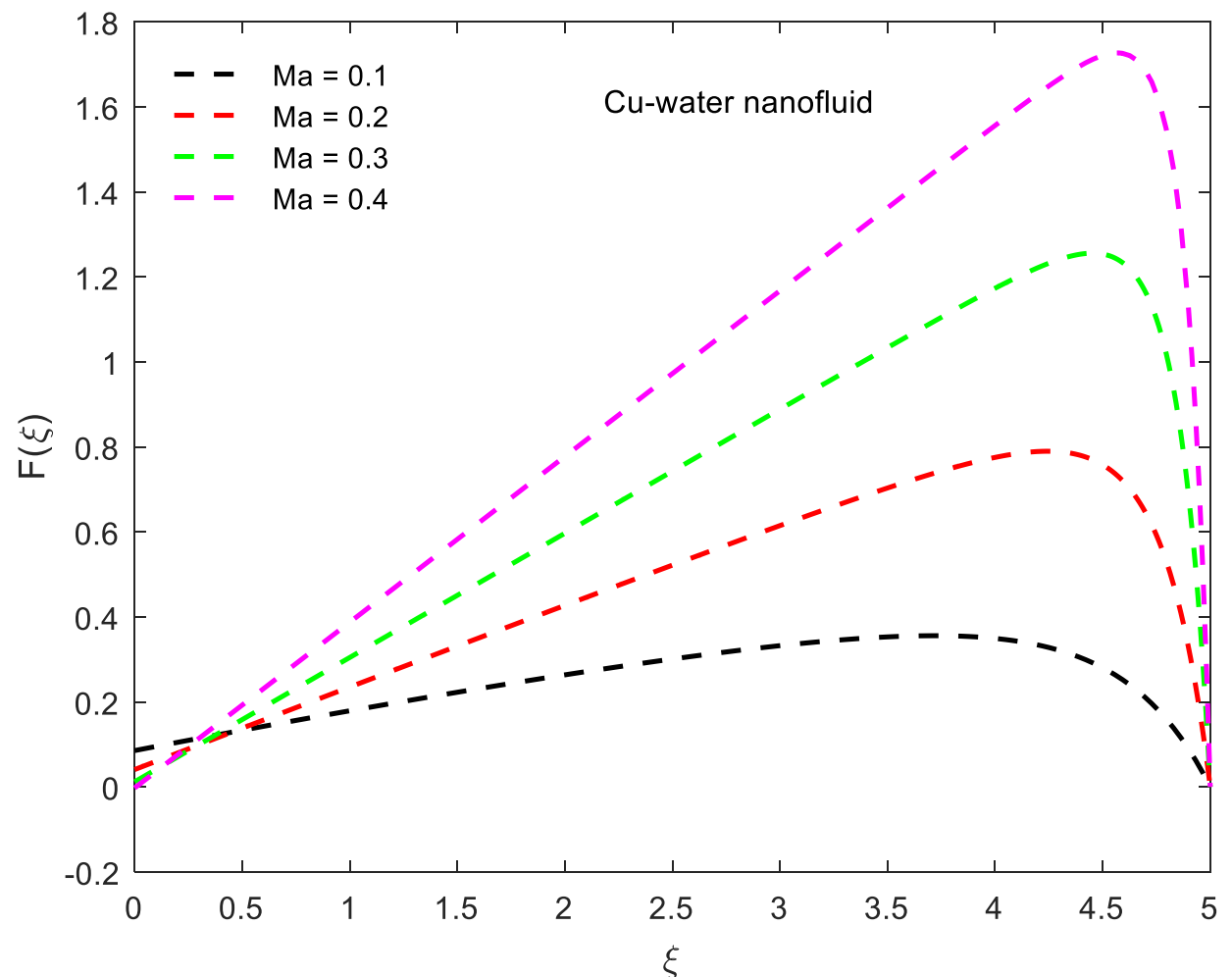

Figure 5: Marangoni parameter impact on $F(\xi)$ for $M=0, P=0, R=0, \operatorname{Pr}=7, \phi=0.01$ 


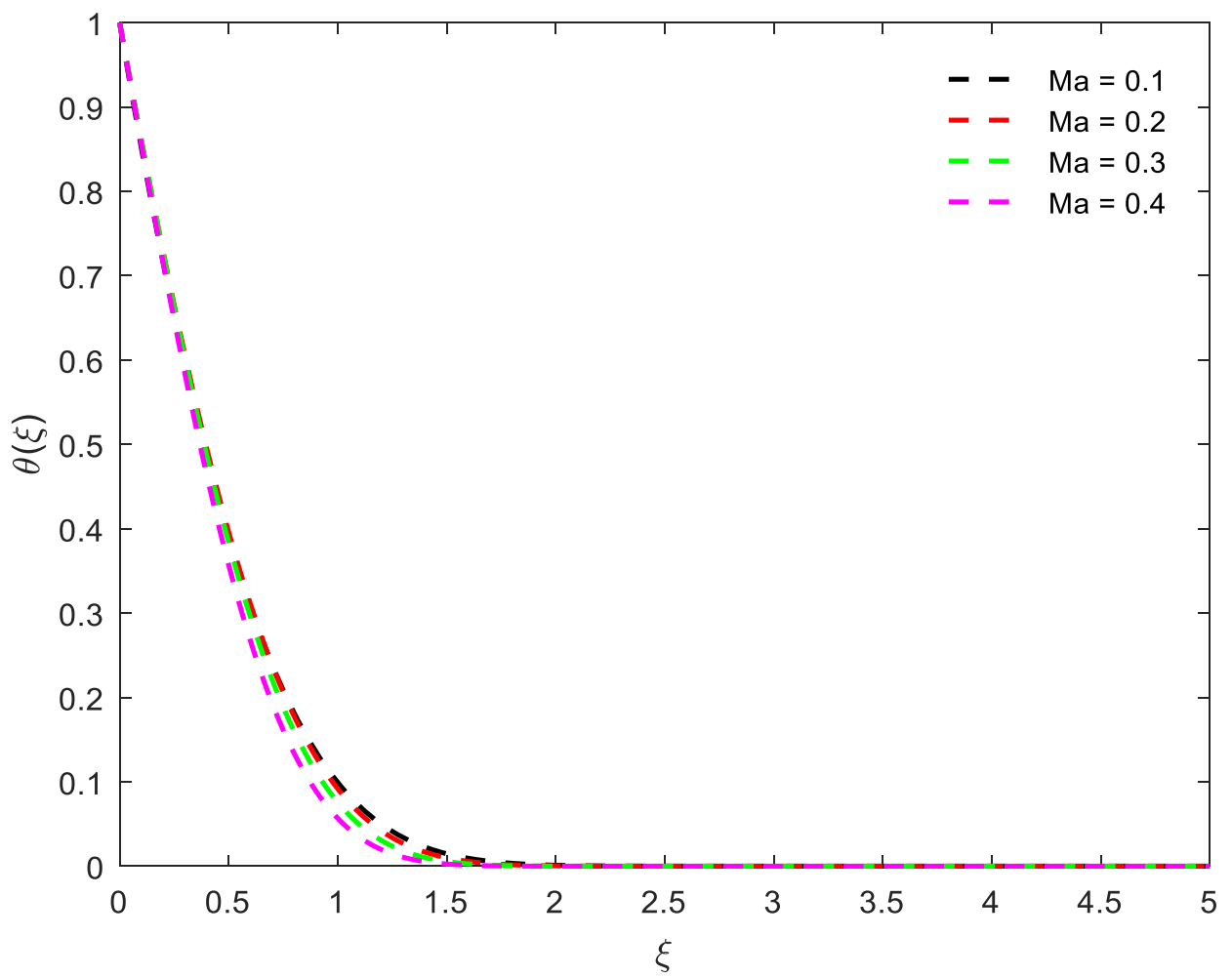

Figure 6: Marangoni parameter impact on $\theta(\xi)$ for $M=0, P=0, R=0, \operatorname{Pr}=7, \phi=0.01$

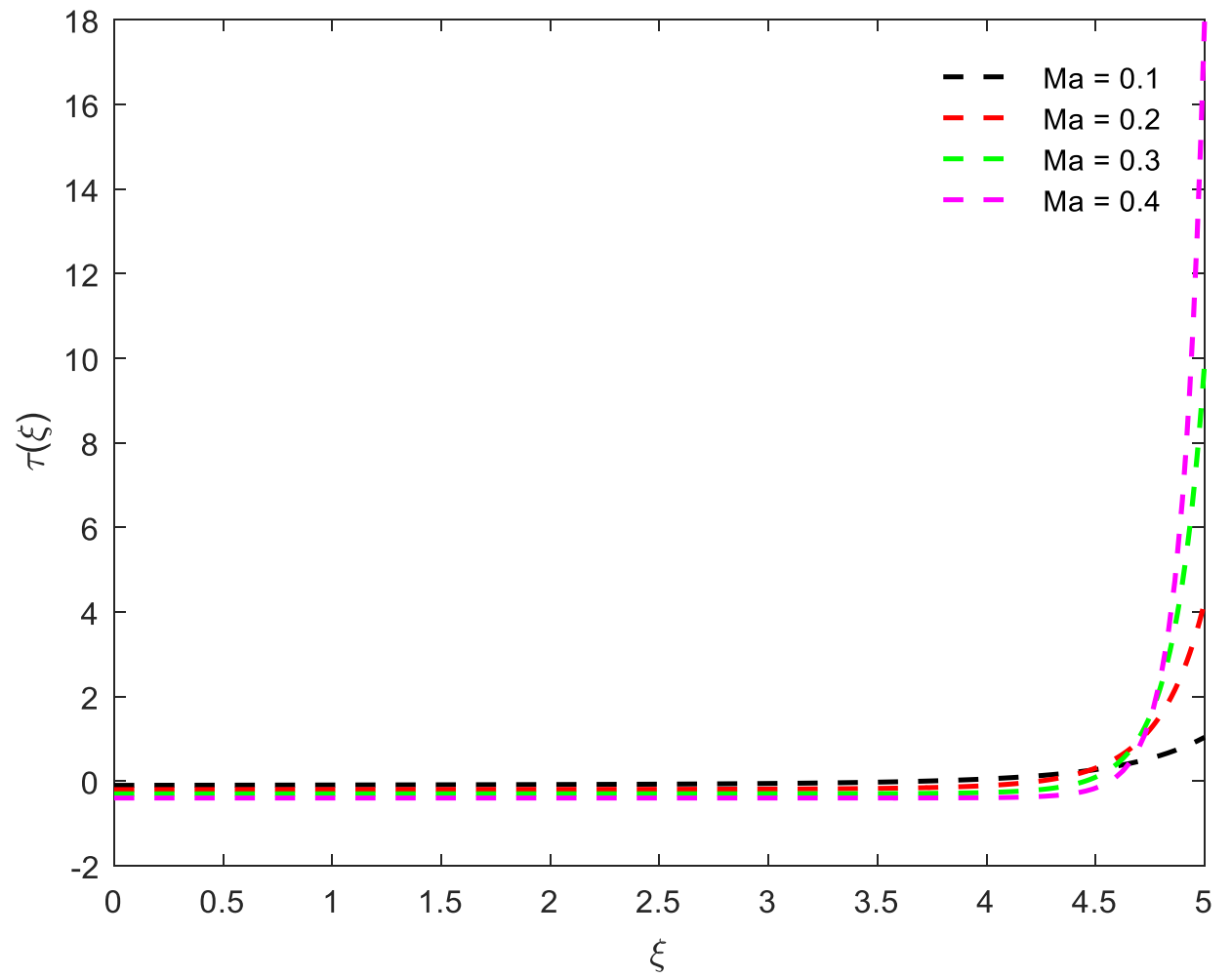

Figure 7: Marangoni parameter impact on $\tau(\xi)$ for $M=0, P=0, R=0, \operatorname{Pr}=7, \phi=0.01$ 


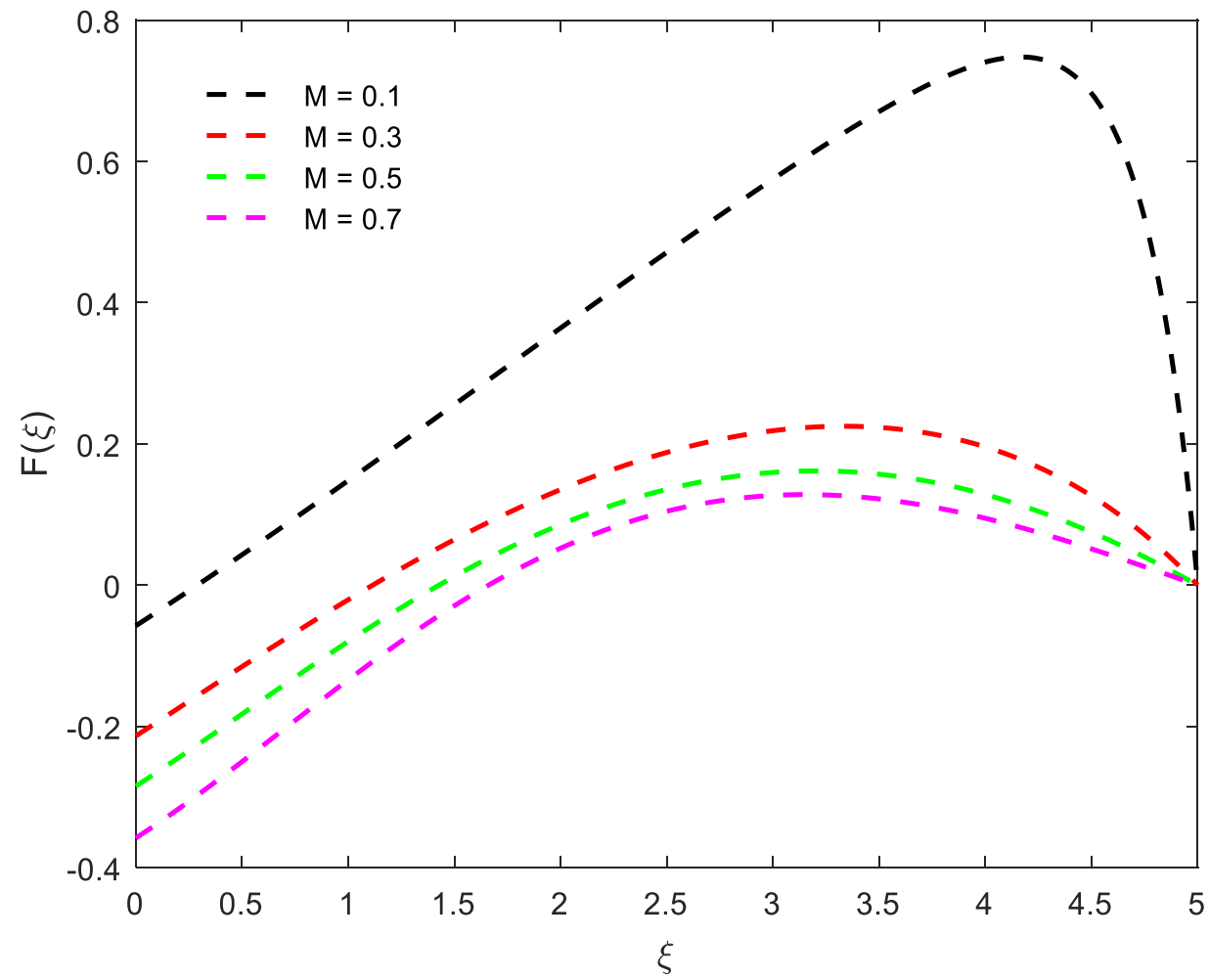

Figure 8: Magnetic term impact on $F(\xi)$ for $M a=0.2, P=0, R=0, \operatorname{Pr}=7, \phi=0.01$

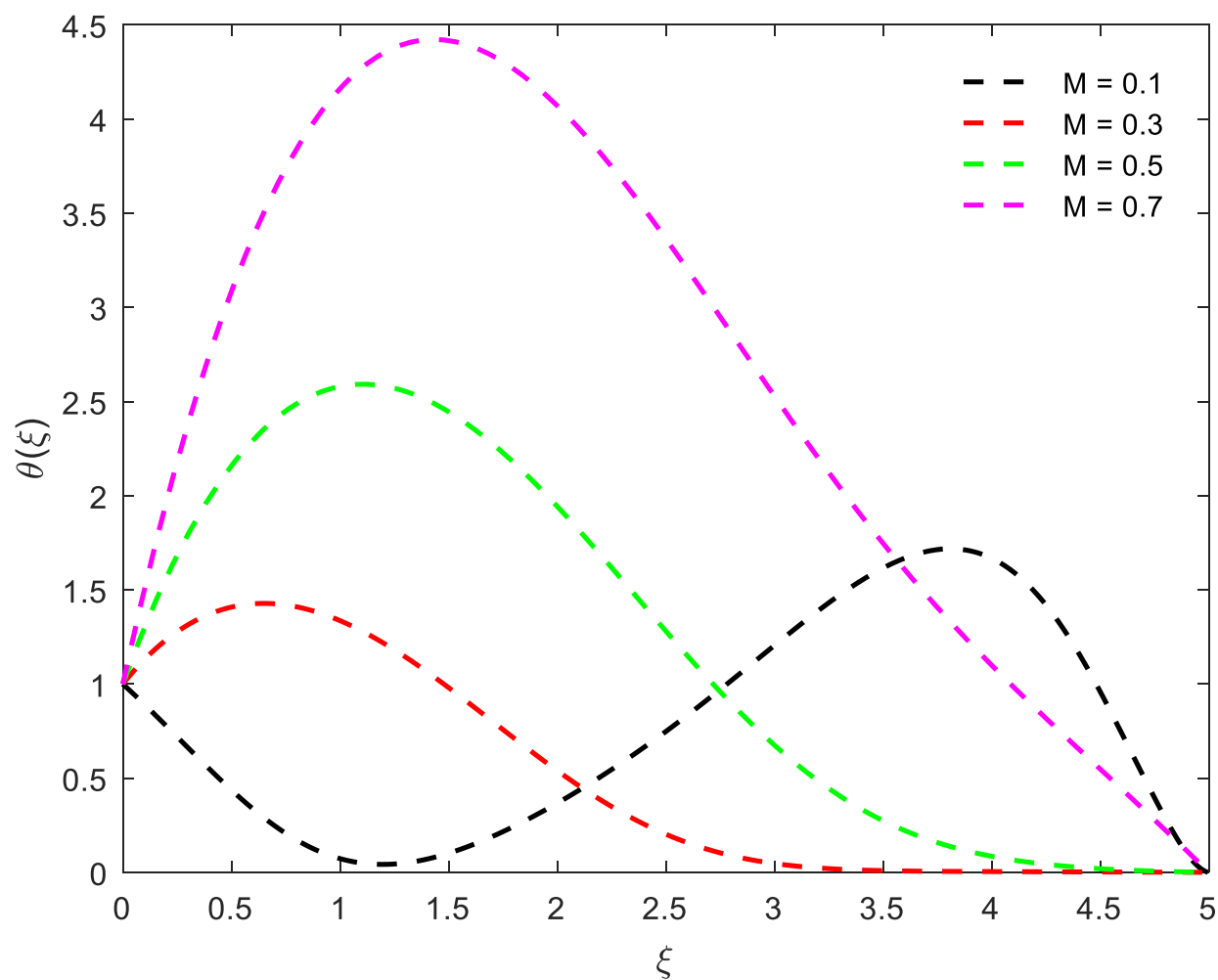

Figure 9: Magnetic term impact on $\theta(\xi)$ for $M a=0.2, P=0, R=0, \operatorname{Pr}=7, \phi=0.01$ 


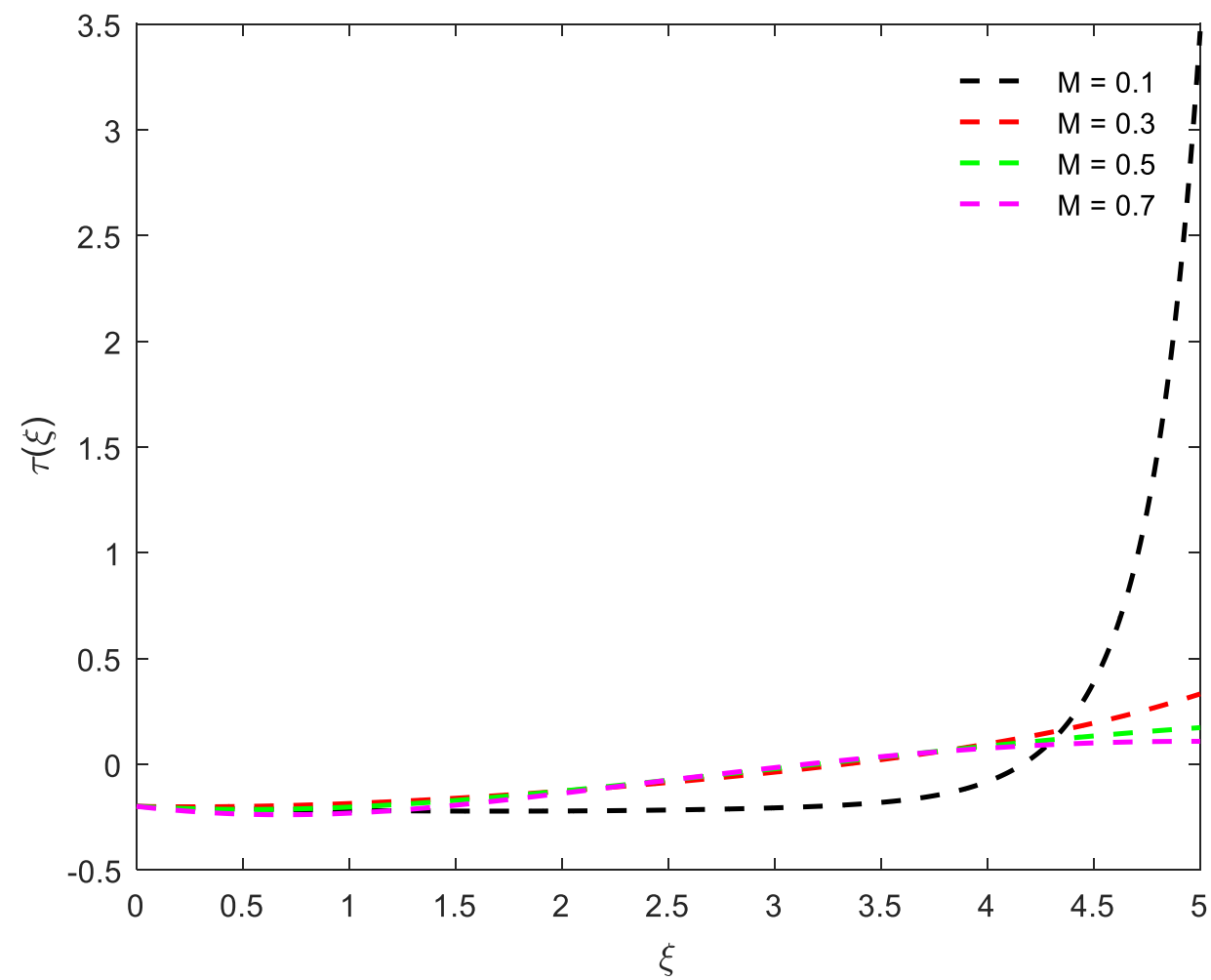

Figure 10: Magnetic term impact on $\tau(\xi)$ for $M a=0.2, P=0, R=0, \operatorname{Pr}=7, \phi=0.01$

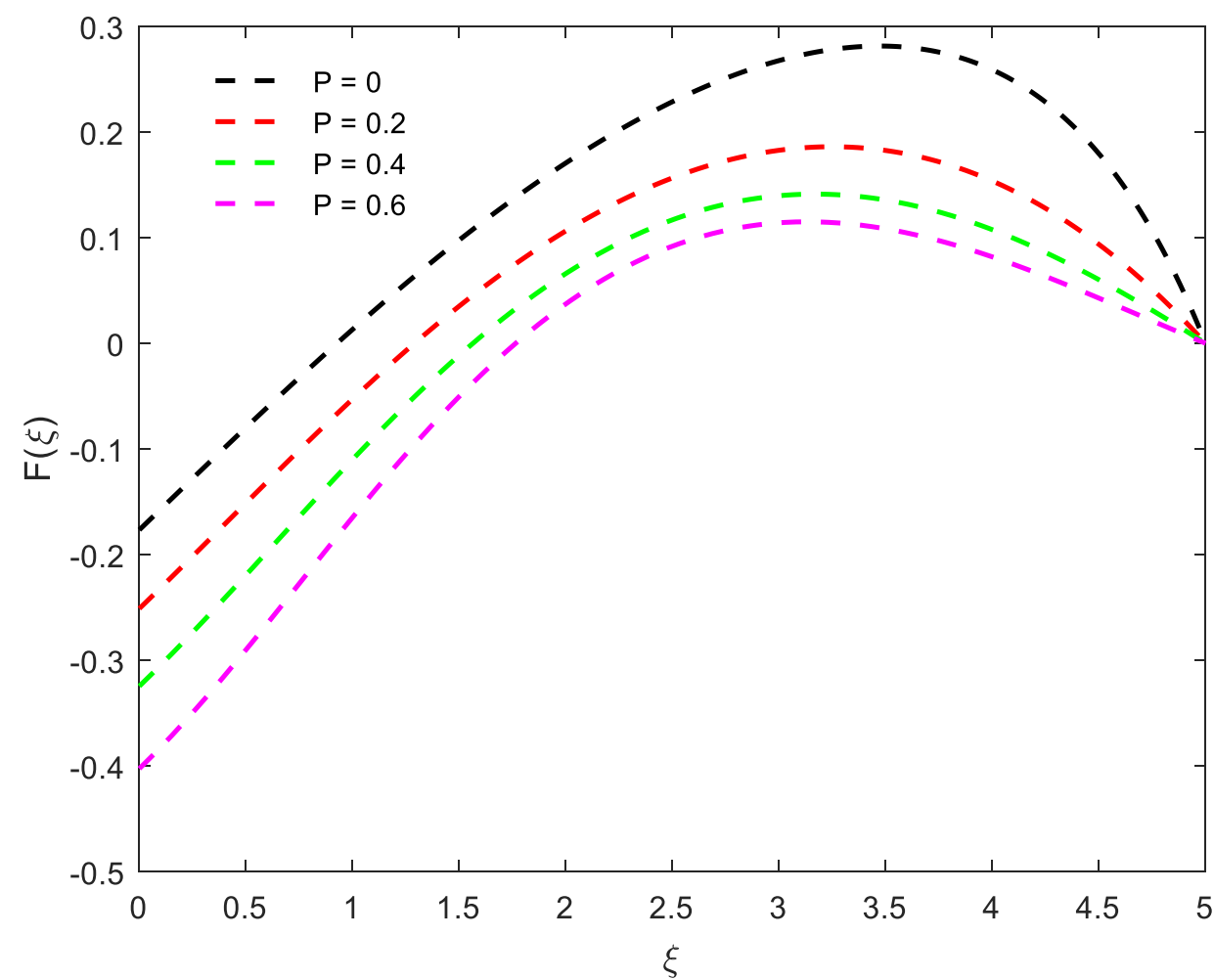

Figure 11: Permeability effect on $F(\xi)$ for $M=0.1, M a=0.2, R=0, \operatorname{Pr}=7, \phi=0.01$ 


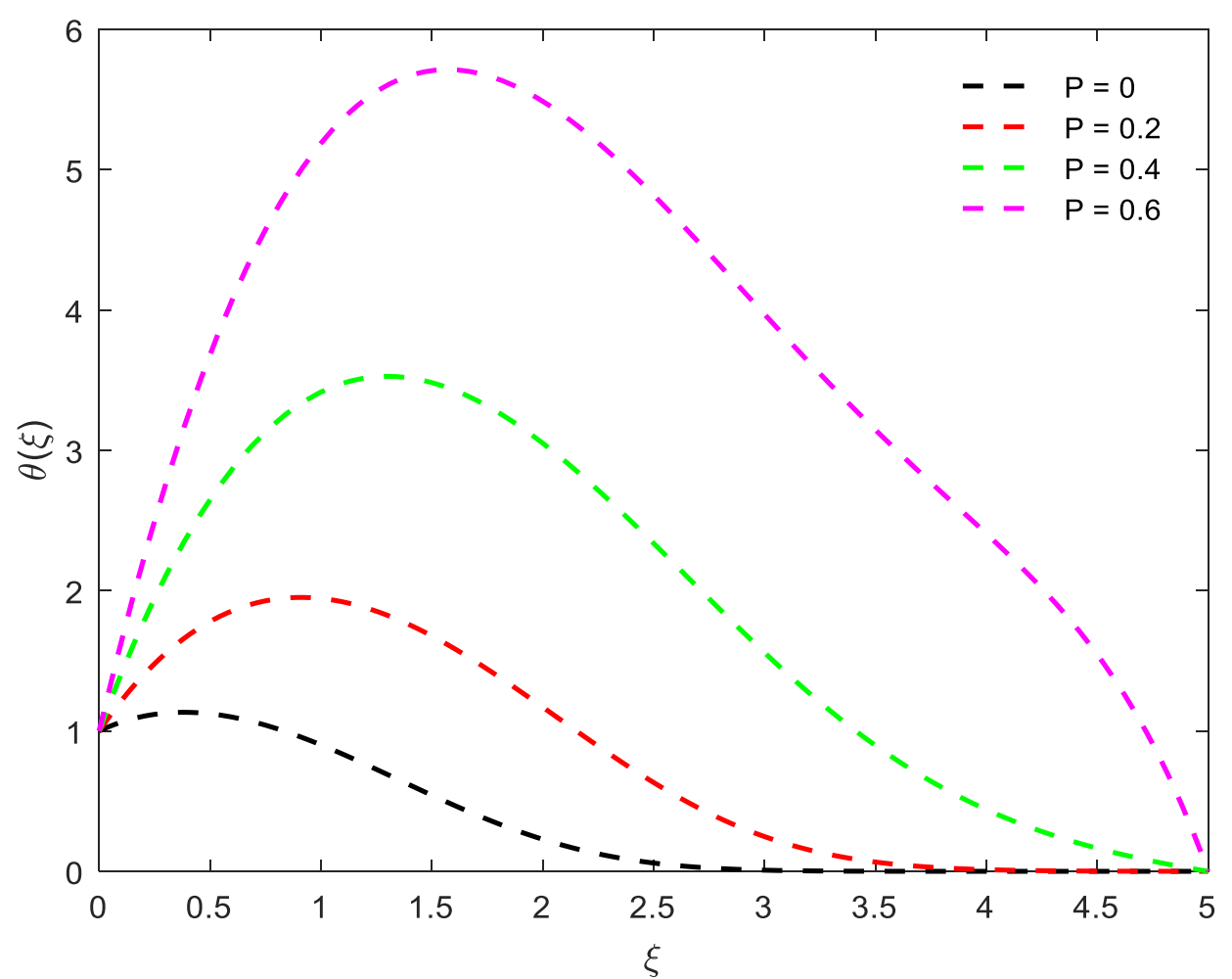

Figure 12: Permeability effect on $\theta(\xi)$ for $M=0.1, M a=0.2, R=0, \operatorname{Pr}=7, \phi=0.01$

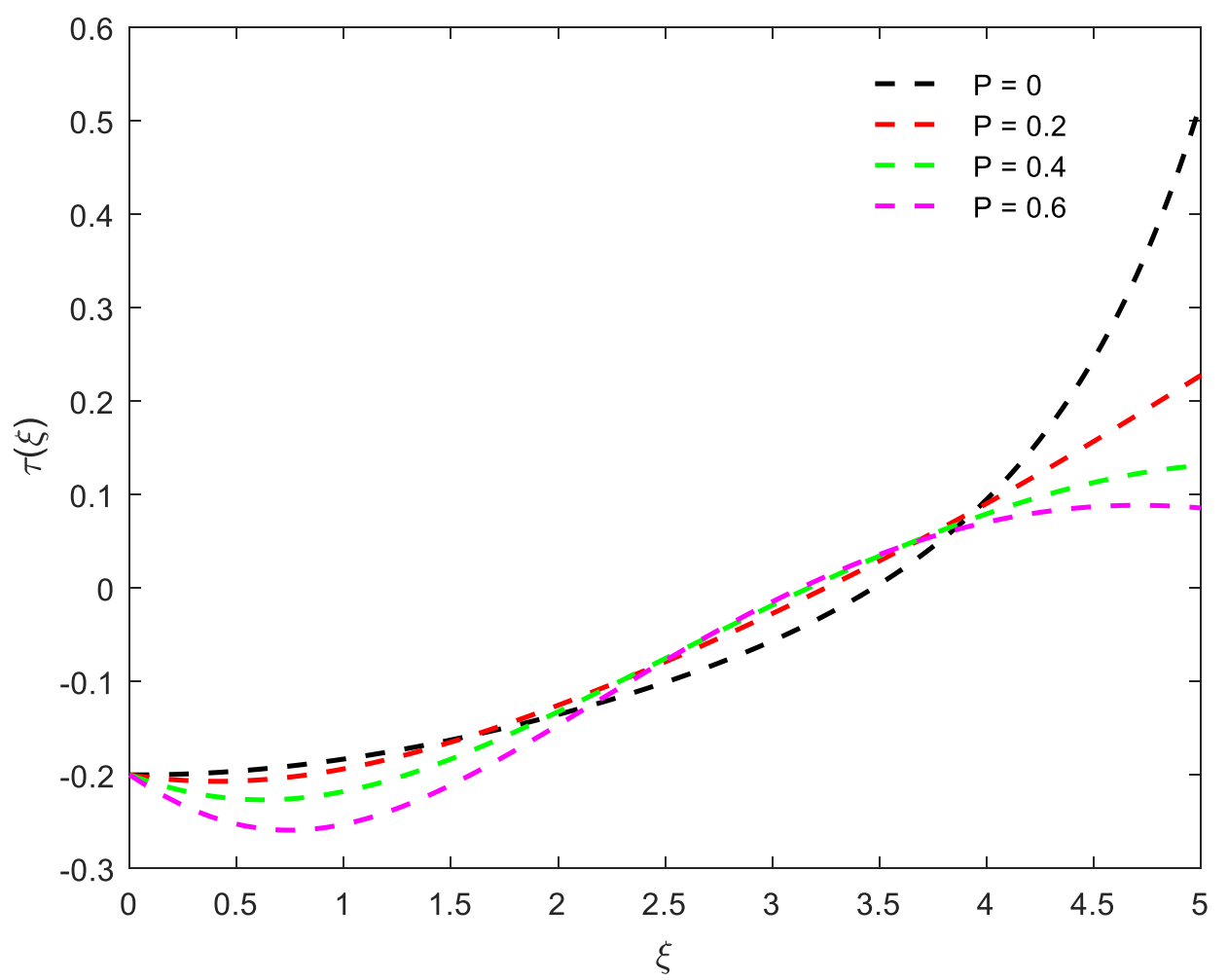

Figure 13: Permeability effect on $\tau(\xi)$ for $M=0.1, M a=0.2, R=0, \operatorname{Pr}=7, \phi=0.01$ 


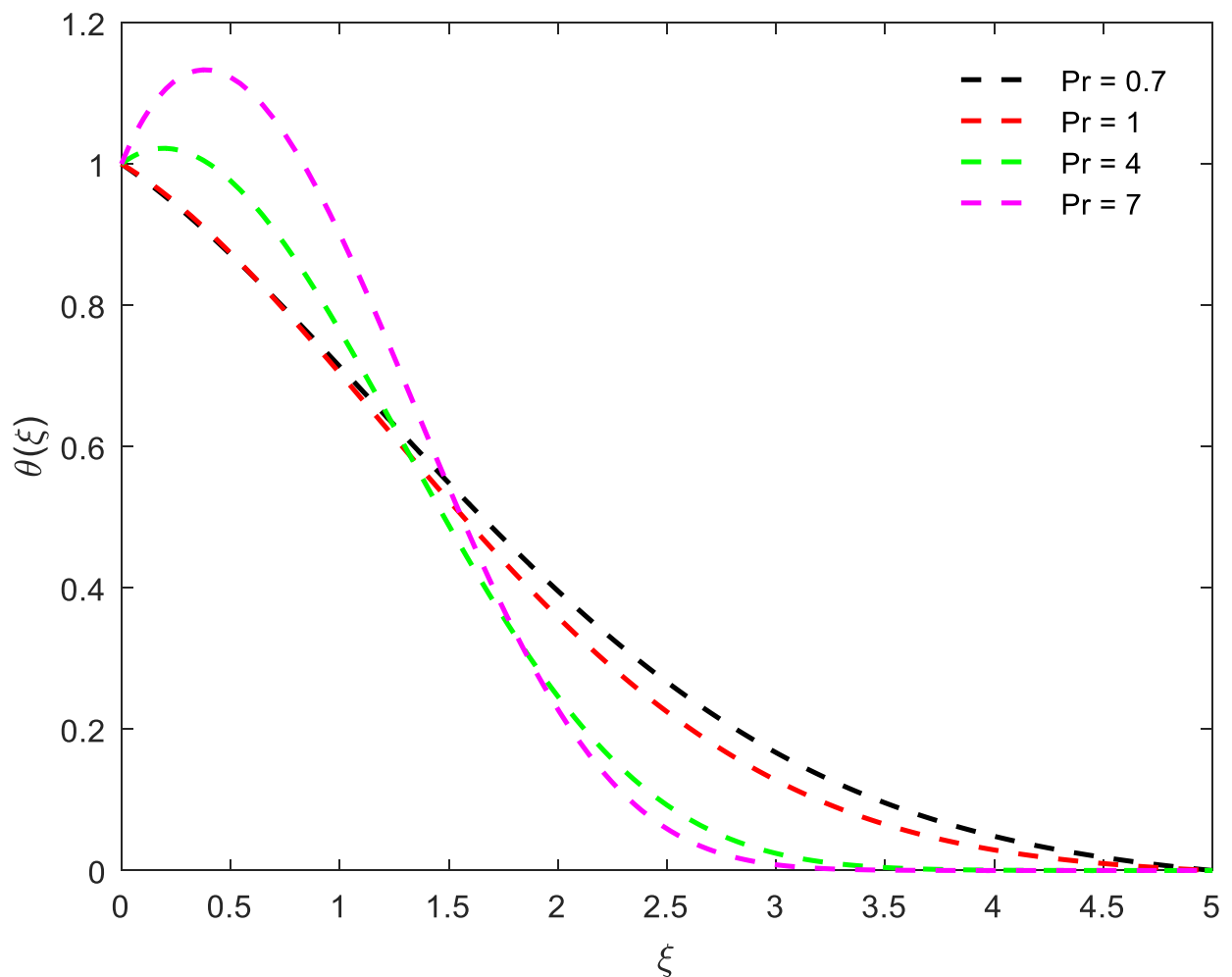

Figure 14: Prandtl number influence on $\theta(\xi)$ for $M=0.1, M a=0.2, P=0, R=0, \phi=0.01$

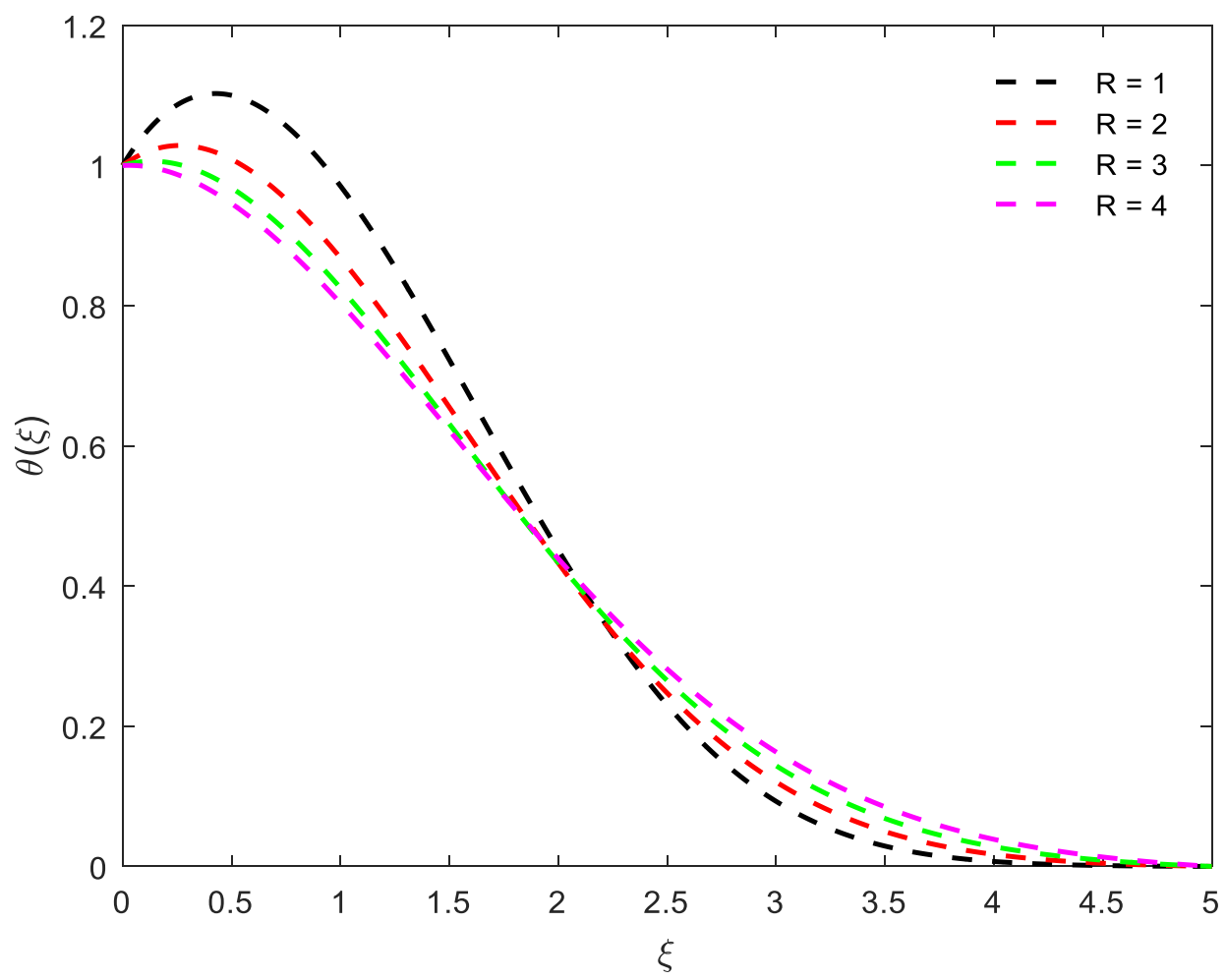

Figure 15: Radiation-conduction term effect on $\theta(\xi)$ for $M=0.1, M a=0.2, P=0, R=0, \phi=0.01$ 


\section{CONCLUSION}

We investigate the thermal radiation and porosity impact on axisymmetric flow of Marangoni fluid with Cu-water nanofluid from a disk under the action of an axial static magnetic field in present study. The semi-analytical Adomain Decomposition solutions is obtained, these simulations have shown that:

$>$ Wall temperature gradient increases and rises for respective variation in $R$ and temperature boosts further from the disk surface.

$>$ Flow is accelerated as temperature diminishes with rise in Marangoni term.

$>$ Increasing permeability (Darcy) term, temperatures are elevated due to the decrease in porous medium permeability i.e. presence of more solid fibers which encourages thermal conduction.

> Strong damping in the velocity field is induced with increasing permeability (Darcy) parameter due to the elevation in Darcian porous bulk matrix impedance.

$>$ The magnetic field suppresses the velocity (generating a weak back flow near the disk surface) but enhances temperature. Robust axial magnetic field also damps temperature oscillations computed at weaker magnetic field strengths.

$>$ Increasing Prandtl number induces a temperature overshoot near the disk surface but thereafter reduces temperatures in the regime.

Rapidly convergent and accurate solutions are achieved with both ADM and GDQ methods and these seminumerical techniques hold excellent promise for simulating more complex Marangoni magnetohydrodynamic nanofluid flows of relevance to coating and materials processing systems including non-Darcy transport Umavathi and Bég [42].

\section{ACKNOWLEDGEMENTS}

The third $(\mathrm{OAB})$ and fourth $(\mathrm{TAB})$ authors would like to express their deep gratitude to Miss Sootina Bég (August $14^{\text {th }} 2008$ - February $22^{\text {nd }} 2020$ ) for her wonderful companionship; she will be greatly missed and never forgotten.

\section{REFERENCES}

[1] Ma N, Walker J, Bliss D (1998) Forced convection during liquid encapsulated crystal growth with an axial magnetic field. ASME J Fluids Eng, 120(4): 844-850.

[2] Langlois WE, Hjellming LN, Walker JS (1987) Effects of the finite electrical conductivity of the crystal on hydromagnetic Czochralski flow. J Crystal Growth, 83(1): 51-61. 
[3] Lin Y, Zheng L, Zhang X (2013) Magnetohydrodynamics thermocapillary Marangoni convection heat transfer of power-law fluids driven by temperature gradient. ASME J Heat Transf, 135(5): 051702. https://doi.org/10.1115/1.4023394.

[4] Rudraiah N, Venkatachalappa M, Subbaraya CK (1995) Combined surface tension and buoyancy-driven convection in a rectangular open cavity in the presence of a magnetic field. Int J Non-Linear Mech, 30(5): 759-770.

[5] Jana S, Dost S, Kumar V, Durst F (2006) A numerical simulation study for the Czochralski growth process of Si under magnetic field. Int J Eng Sci, 44 (8-9): 554-573.

[6] Zueco J, Anwar Bég O (2010) Network numerical simulation of hydromagnetic Marangoni mixed convection boundary layers. Chem Eng Commun, 198(4): 552-571.

[7] Witkowski LM, Walker JS (2001) Flow driven by Marangoni convection and rotating magnetic field in a floating-zone configuration. Magnetohydrodynamics, 37: 112-118.

[8] Hajabdollahi F, Premnath KN (2018) Thermocapillary convection due to imposed interfacial heating in the presence of magnetic field. J Eng Math, 108: 37-52.

[9] Flamant Q, Clarke DR (2019) Opportunities for minimizing radiative heat transfer in future thermal and environmental barrier coatings. Scripta Materialia, 173: 26-31.

[10] Anwar Bég O, Zueco J, López-Ochoa LM (2011) Network numerical analysis of optically-thick hydromagnetic slip flow from a porous spinning disk with radiation flux, variable thermophysical properties and surface injection effects. Chem Eng Commun, 198(3): 360-384.

[11] Ganesh NV, Kameswaran PK, Al-Mdallal, Qasem M, Hakeem AKA, Ganga B (2018) Non-linear thermal radiative Marangoni boundary layer flow of gamma $\mathrm{Al}_{2} \mathrm{O}_{3}$ nanofluids past a stretching sheet. J Nanofluids, 7(5): 944-950.

[12] Lin Y, Zheng L, Zhang X (2014) Radiation effects on Marangoni convection flow and heat transfer in pseudo-plastic non-Newtonian nanofluids with variable thermal conductivity. Int J Heat and Mass Transf, 77: 708-716.

[13] Mahanthesh B, Gireesha BJ, Animasaun IL, Taseer M, Shashikumar NS (2017) Marangoni convective MHD flow of SWCNT and MWCNT nanoliquids due to a disk with solar radiation and irregular heat source. Physica E: Low-dimensional Systems and Nanostructures, 94(8): 25-30.

[14] Bayazitoglu Y, Lam TT (1987) Marangoni convection in radiating fluids. ASME J Heat Transf, 109(3): 717-721. 
[15] Lin Y, Zheng L (2015) Marangoni boundary layer flow and heat transfer of Copper-water nanofluid over a porous medium disk. AIP Adv, 5: Article ID:107225. https://doi.org/10.1063/1.4934932.

[16] Adomian G (1994) Solving Frontier problems of Physics: The decomposition method. Kluwer Academic Publishers, USA.

[17] Shu C, Richards BE (1992) Application of generalized differential quadrature to solve two-dimensional incompressible Navier-Stokes equations. Int J Numer Meth Fluids, 15(7): 791-798.

[18] Zhang Z, Peng B, Ji X, Pei K, Chan PKL (2017) Marangoni-effect-assisted bar-coating method for highquality organic crystals with compressive and tensile strains. Adv Functional Mat, 27: Article ID:1703443 (37 pages). htpps://doi.org/10.1002/adfm.201703443.

[19] Anwar Bég O, Ferdows M, Islam MS, Islam MN (2014) Numerical simulation of Marangoni magnetohydrodynamic bio-nanofluid convection from a non-isothermal surface with magnetic induction effects: a bio-nanomaterial manufacturing transport model. J Mech Med Biol, 14(3): 1450039.11450039.32 .

[20] Bochner de Araujo S, Carvalho MS (2017) Sedimentation and Marangoni stress in slot coating flow of particle suspension. J Non-Newtonian Fluid Mech, 247: 53-61.

[21] Anwar Beg O, Islam B, Shamshuddin MD, Beg TA (2019) Computational fluid dynamics analysis of moisture ingress in aircraft structural composite materials. Arabian J Sci Eng, 44(9): 7809-7831.

[22] Sheremet MA, Pop I (2019) Marangoni natural convection in a cubical cavity filled with a nanofluid. J Therm Anal Calorimetry, 135: 357-369.

[23] Dogonchi AS, Chamkha AJ, Ganji DD (2019) A numerical investigation of magneto-hydrodynamic natural convection of $\mathrm{Cu}$-water nanofluid in a wavy cavity using CVFEM. J Therm Anal Calorimetry, 135: 25992611.

[24] Tiwari RJ, Das MK (2007) Heat transfer augmentation in a two-sided lid-driven differentially heated square cavity utilizing nanofluids. Int J Heat Mass Transf, 50: 2002-2018.

[25] Aneja M, Sapna S, Kuharat S, Anwar Bég O (2020) Computation of electroconductive gyrotactic bioconvection under non-uniform magnetic field: Simulation of smart bio-nanopolymer coatings for solar energy. Int J Modern Physics B, (22 pages). https://doi.org/ 10.1142/S0217979220500289

[26] Kuharat S, Anwar Bég O (2019) Computational fluid dynamics simulation of a nanofluid-based annular solar collector with different metallic nanoparticles. Heat and Mass Transf. Res. J, 3(1):1-23.

[27] Rosseland S (1936) Theoretical astrophysics, Oxford University, New York, USA. 
[28] Brinkmann HC (1952) The viscosity of concentrated suspensions and solutions, The J Chem Phys, 20(4): $571-581$.

[29] Aski FS, Nasirkhani SJ, Mohammadian E, Asgari A (2014) Application of Adomian decomposition method for micropolar flow in a porous channel. Prop Power Res, 3(1): 15-21.

[30] Anwar Bég O, Tripathi D, Sochi T, Gupta PK (2015) Adomian decomposition method (ADM) simulation of magneto-bio-tribological squeeze film with magnetic induction effects. J Mech Med Biol, 15: 1550072.1-1550072.23.

[31] Anwar Bég O, Mabood F, Islam MN (2015) Homotopy simulation of nonlinear unsteady rotating nanofluid flow from a spinning body. Int J Eng Math, 2015: Article ID:272079. https://doi.org/10.1155/2015/272079

[32] Manzoor N, Maqbool K, Anwar Bég O, Shaheen S (2018) Adomian decomposition solution for propulsion of dissipative magnetic Jeffrey biofluid in a ciliated channel containing a porous medium with forced convection heat transfer. Heat Transfer - Asian Res, 48(2): 556-581.

[33] Shamshuddin MD, Mishra SR, Anwar Beg O, Kadir A (2019) Adomain decomposition method simulation of Von Karman swirling bioconvection nanofluid flow. J Cent South Univ-Sci Tech Mining and Metallurgy, 26(10): 2797-2813.

[34] Vasu B, Ray AK, Anwar Bég O, Gorla RSR, Murthy PVSN (2019) Magneto-bioconvection flow of a Casson thin film with nanoparticles over an unsteady stretching sheet: HAM and GDQ computation. Int J Numer Methods Heat Fluid Flow, 29(11): 4277-4309.

[35] Shu C, Chew YT, Richards BE (1995) Generalized differential-integral quadrature and their application to solve boundary layer equations. Int J Numer Methods in Fluids, 21: 723- 733.

[36] Anwar Bég O (2012) Numerical methods for multi-physical magnetohydrodynamics. Chapter 1, pp. 1-112, New Developments in Hydrodynamics Research (M. J. Ibragimov and M. A. Anisimov, Eds.,), Nova Science, New York, USA.

[37] Anwar Bég O, Kuharat S, Ferdows M, Das M, Kadir A, Shamshuddin MD (2019) Magnetic nano-polymer flow with magnetic induction and nanoparticle solid volume fraction effects: solar magnetic nano-polymer fabrication simulation. Proc IMechE J Nanoeng Nanomat Nanosyst, 233(1): 27-45.

[38] Choi SUS, Zhang ZG, Yu W, Lockwood FE, Grulke EA (2001) Anomalously thermal conductivity enhancement in nanotube suspensions. Appl Phys Lett, 79: 2252-2254.

[39] Vafai K (1984) Convective flow and heat transfer in variable-porosity media. J Fluid Mech, 147: 233-259. 
[40] Vafai K, Tien CL (1981) Boundary and inertia effects on flow and heat transfer in porous media. Int J Heat Mass Transf, 24: $195-203$.

[41] Anwar Bég O, Prasad VR, Vasu B, Gorla RSR (2017) Computational modelling of magnetohydrodynamic convection from a rotating cone in orthotropic Darcian porous media. J Braz Soc Mech Sci Eng, 39: 20352054.

[42] Umavathi JC, Anwar Bég O (2019) Numerical study of double-diffusive dissipative reactive convective flow in an open vertical duct containing a non-Darcy porous medium with Robin boundary conditions. J Eng Math, 119: 135-147. 